\title{
A putative AOP for pneumonia related to COVID-19
}

\author{
Mathieu Vinken ${ }^{1}[$
}

Received: 17 June 2020 / Accepted: 15 July 2020 / Published online: 20 July 2020

c) Springer-Verlag GmbH Germany, part of Springer Nature 2020

According to the coronavirus disease 2019 (COVID-19) case tracker of the Johns Hopkins University, more than 13 million people around the world have been infected with severe acute respiratory syndrome coronavirus 2 (SARS-CoV-2) and as much as 575,000 COVID-19-related deaths have been recorded by mid-July 2020 (https://coronavirus.jhu. edu/). One of the most frequent complications of COVID19 is pneumonia, which underlies symptoms like cough and shortness of breath. The clinical course of COVID-19 pneumonia exhibits a broad spectrum of severity and progression patterns. Severely ill patients can rapidly progress to acute respiratory distress syndrome (ARDS) within a week. ARDS is observed in 17-29\% of hospitalized patients with a global mortality of approximately 5.4\% for all COVID- 19 cases (Tu et al. 2020). Given the current worldwide intensive research efforts, the molecular mechanisms of pneumonia linked to COVID-19 are being elucidated at high pace. A practical tool to capture and visualize mechanisms of adversity, such as toxicity and disease, is the adverse outcome pathway (AOP) construct (Ankley et al. 2010). An AOP starts by the interaction of a foreign entity with a biological system, a process called the molecular initiating event (MIE). Activation of the MIE elicits a chain of key events (KEs) at different levels of biological organization, including at the cellular, tissue and organ level, eventually resulting in the actual adverse outcome (AO), usually occurring at the organism level (Vinken et al. 2017). The Organization for Economic Co-operation and Development (OECD), in collaboration with a number of other agencies, has established an electronic repository for AOPs, called the AOP Wiki. At present, the AOP Wiki contains more than 300 AOPs for a multitude of adverse effects, including an AOP on acute respiratory distress associated with COVID-19 (https://aopwi ki.org/aops/320). This AOP was mainly designed to allow

Mathieu Vinken

mathieu.vinken@vub.be

http://www.mathieuvinken.com

1 Department of In Vitro Toxicology, Vrije Universiteit Brussel, Laarbeeklaan 103, 1090 Brussels, Belgium insight into the mechanism of the cellular entry of viral particles and to support vaccine development. In order to further encourage research in this direction, an updated version of the putative AOP for pneumonia linked to COVID-19 is proposed, which encompasses new knowledge that is rapidly accumulating (Fig. 1). One of the major MIEs in this AOP is the binding of SARS-CoV-2 to the angiotensin-converting enzyme 2 (ACE2) receptor at the plasma membrane surface of type II pneumocytes lining the alveoli in lung. This binding occurs through subunit $\mathrm{S} 1$ of the SARS-CoV-2 surface spike glycoprotein $\mathrm{S}$, with the latter being primed by cellular transmembrane serine protease 2 (TMPRSS2) (Xiao et al. 2020). Upon entry, which involves internalization of both SARS-CoV-2 and the ACE2 receptor, the virus replicates by making use of the ribosomes of the host cell and by relying on the actions of RNA replicase. Following assembly of the de novo synthetized viral proteins, the newly formed SARSCoV-2 is released and propagates infection (Tu et al. 2020). The process of viral replication triggers an inflammatory response accompanied by the recruitment of macrophages. This most likely involves activation of the NOD-like receptor family pyrin domain-containing 3 (NLRP3) inflammasome, which facilitates the production of pro-inflammatory cytokines, including interleukin $1 \beta$ and interleukin 18 . NLRP3 induces the formation of gasdermin-D pores on the plasma membrane surface, causing secretion of interleukin $1 \beta$ and interleukin 18 as well as the influx of water into the cytosol, which in turn leads to cell swelling and subsequent rupture or so-called pyroptosis (Yap et al. 2020). The released pro-inflammatory cytokines, along with changes in angiotensin homeostasis, compromise the structure and functionality of surrounding endothelial cells in alveolar vasculature. In particular, permeability becomes increased and goes hand in hand with endothelial fluid leakage and the occurrence of edema (Leisman et al. 2020). Simultaneously, released pro-inflammatory cytokines stimulate the generation of reactive oxygen and nitrogen species, and thus the onset of oxidative stress (Ntyonga-Pono 2020). In parallel, several enzyme systems, in particular proteases, are activated, which damage cellular architecture. Collectively, 


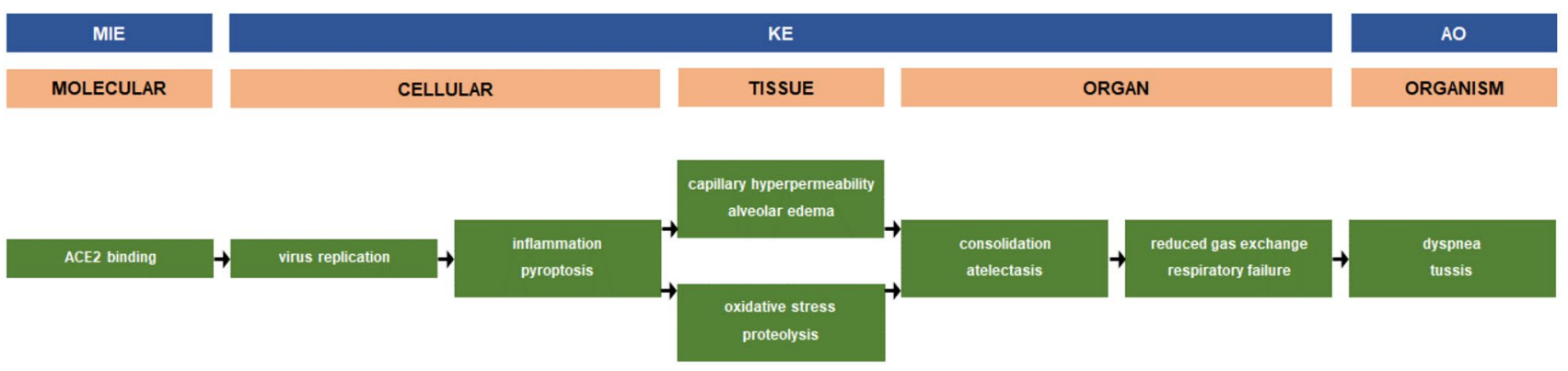

Fig. 1 A putative AOP for pneumonia linked to COVID-19

these deteriorating events instigate consolidation, denoting the replacement of air in the alveoli by transudate, pus, blood or cells, which presents as the typical "white lungs" during radiography. This may or may not coincide with collapse of the lung or so-called atelectasis (Fu et al. 2020) The outcome of this is reduced or even absent gas exchange and hence respiratory failure (Gallelli et al. 2020). This is ultimately manifested clinically as dry cough and shortness of breath, also called tussis and dyspnea, respectively (Öztürk 2020), being considered as the actual adverse outcome (AO).

It should be stressed that this proposed AOP must be considered with caution, as it has not been assessed according to the tailored Bradford-Hill criteria to evaluate biological plausibility, essentiality and empirical support (Becker et al. 2015). Indeed, the relevance and sequence of some KEs as well as their links may not be fully substantiated by current information. Inherent to their dynamic nature, AOPs should be updated and finetuned as soon as new relevant data become available. This certainly holds true for the AOP for pneumonia linked to COVID-19. In fact, the proposed AOP should eventually evolve into an AOP network that considers all clinically pertinent MIEs and AOs. A number of efforts towards this goal are currently ongoing, including at the European Commission's Joint Research Center (JRC). Such AOP network should comprise the mechanisms driving the multi-organ failure frequently observed in severe COVID19 patients, for which the causes (i.e. MIEs) are as yet not entirely clear or delineated. Thus, liver failure may be caused by the direct binding and actions of SARS-CoV-2 in hepatocytes or cholangiocytes, but could also be an indirect consequence of the systemic inflammatory response syndrome associated with COVID-19. In addition, liver failure may result from hepatic injury caused by drugs administered to COVID-19 patients (Alqahtani and Schattenberg 2020). The future COVID-19 AOP network could also cover the different steps of virus infection and, especially, replication, now captured in a single KE. This could be of specific relevance for the development of novel drugs and vaccines, which typically target this process. The overall COVID-19 AOP network could serve a number of purposes, a prominent one being disease modeling. In this respect, an appealing opportunity lies ahead to generate animal-free new approach methodologies (NAMs) that allow to study particular aspects of the COVID-19 spectrum (Busquet et al. 2020). By optimally combining these NAMs, mechanistically anchored in the AOP network, further insight into COVID-19 pathogenesis will be gained, which in turn will open perspectives for setting up targeted clinical treatment strategies.

\section{Compliance with ethical standards}

Conflict of interest The author declares that he has no conflict of interest.

\section{References}

Alqahtani SA, Schattenberg JM (2020) Liver injury in COVID-19: the current evidence. United Eur Gastroenterol J 8:509-519

Ankley GT, Bennett RS, Erickson RJ, Hoff DJ, Hornung MW, Johnson RD, Mount DR, Nichols JW, Russom CL, Schmieder PK, Serrrano JA, Tietge JE, Villeneuve DL (2010) Adverse outcome pathways: a conceptual framework to support ecotoxicology research and risk assessment. Environ Toxicol Chem 29:730-741

Becker RA, Ankley GT, Edwards SW, Kennedy SW, Linkov I, Meek B, Sachana M, Segner H, Van Der Burg B, Villeneuve DL, Watanabe H, Barton-Maclaren TS (2015) Increasing scientific confidence in adverse outcome pathways: application of tailored Bradford-Hill considerations for evaluating weight of evidence. Regul Toxicol Pharmacol 72:514-537

Busquet F, Hartung T, Pallocca G, Rovida C, Leist M (2020) Harnessing the power of novel animal-free test methods for the development of COVID-19 drugs and vaccines. Arch Toxicol 94:2263-2272

Fu F, Lou J, Xi D, Bai Y, Ma G, Zhao B, Liu D, Bao G, Lei Z, Wang M (2020) Chest computed tomography findings of coronavirus disease 2019 (COVID-19) pneumonia. Eur Radiol. https://doi. org/10.1007/s00330-020-06920-8

Gallelli L, Zhang L, Wang T, Fu F (2020) Severe acute lung injury related to COVID-19 infection: a review and the possible role for escin. J Clin Pharmacol 60:815-825

https://aopwiki.org/aops/320 Accessed 14 July 2020

https://coronavirus.jhu.edu/ Accessed on 14 July 2020 
Leisman DE, Deutschman CS, Legrand M (2020) Facing COVID-19 in the ICU: vascular dysfunction, thrombosis, and dysregulated inflammation. Intensive Care Med 46:1105-1108

Ntyonga-Pono MP (2020) COVID-19 infection and oxidative stress: an under-explored approach for prevention and treatment? Pan Afr Med J 35:12

Öztürk R (2020) COVID-19: pathogenesis, genetic polymorphism, clinical features and laboratory findings. Turk J Med Sci 50:638-657

Tu YF, Chien CS, Yarmishyn AA, Lin YY, Luo YH, Lin YT, Lai WY, Yang DM, Chou SJ, Yang YP, Wang ML, Chiou SH (2020) A review of SARS-CoV-2 and the ongoing clinical trials. Int J Mol Sci 21:2657

Vinken M, Knapen D, Vergauwen L, Hengstler JG, Angrish M, Whelan M (2017) Adverse outcome pathways: a concise introduction for toxicologists. Arch Toxicol 91:3697-3707
Xiao L, Sakagami H, Miwa N (2020) ACE2: the key molecule for understanding the pathophysiology of severe and critical conditions of COVID-19: demon or angel? Viruses 12:E491

Yap JKY, Moriyama M, Iwasaki A (2020) Inflammasomes and pyroptosis as therapeutic targets for COVID-19. J Immunol 205:307-312

Publisher's Note Springer Nature remains neutral with regard to jurisdictional claims in published maps and institutional affiliations. 